\title{
Neutral Hydrogen Observations of Low Surface Brightness Galaxies around M101 and NGC 5485
}

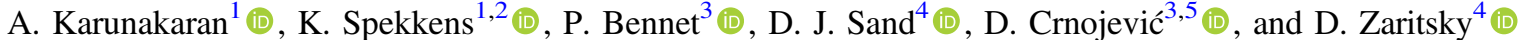 \\ ${ }^{1}$ Department of Physics, Engineering Physics and Astronomy, Queen's University, Kingston, ON K7L 3N6, Canada; a.karunakaran@queensu.ca \\ ${ }^{2}$ Department of Physics and Space Science, Royal Military College of Canada P.O. Box 17000, Station Forces Kingston, ON K7K 7B4, Canada \\ ${ }_{3}^{3}$ Physics \& Astronomy Department, Texas Tech University, Box 41051, Lubbock, TX 79409-1051, USA \\ ${ }^{4}$ Steward Observatory, University of Arizona, 933 North Cherry Avenue, Rm. N204, Tucson, AZ 85721-0065, USA \\ ${ }^{5}$ University of Tampa, 401 West Kennedy Boulevard, Tampa, FL 33606, USA \\ Received 2019 October 11; revised 2019 November 19; accepted 2019 November 21; published 2020 January 3
}

\begin{abstract}
We present atomic hydrogen (H I) observations using the Robert C. Byrd Green Bank Telescope along the lines of sight to 27 low surface brightness (LSB) dwarf galaxy candidates discovered in optical searches around M101. We detect $\mathrm{H}$ I reservoirs in five targets and place stringent upper limits on the remaining 22, implying that they are gaspoor. The distances to our $\mathrm{H}$ I detections range from 7 to $150 \mathrm{Mpc}$, demonstrating the utility of wide-bandpass $\mathrm{H} \mathrm{I}$ observations as a follow-up tool. The systemic velocities of three detections are consistent with that of the NGC 5485 group behind M101, and we suggest that our 15 nondetections with lower distance limits from the optical are associated with and have been stripped by that group. We find that the gas richness of confirmed M101 satellites are broadly consistent with those of the Milky Way satellites, as well as with those of satellites around other hosts of comparable mass, when survey completeness is taken into account. This suggests that satellite quenching and gas stripping proceed similarly around halos of similar mass, in line with theoretical expectations.
\end{abstract}

Unified Astronomy Thesaurus concepts: Dwarf galaxies (416); Low surface brightness galaxies (940); H I line emission (690); Galaxy evolution (594)

\section{Introduction}

Recent improvements in astronomical instrumentation (e.g., Abraham \& van Dokkum 2014; Aihara et al. 2018) and novel image searching algorithms (e.g., Bennet et al. 2017; Müller et al. 2018; Zaritsky et al. 2019) have revitalized studies of the low surface brightness (LSB) universe. Many of these LSB features detected resemble dwarf galaxies (Merritt et al. 2014), while some have extreme properties relative to the high surface brightness galaxy population (e.g., van Dokkum et al. 2015).

Wide-field LSB searches for faint companions of nearby galaxies allow comparisons with Local Group satellite populations, and there have been concerted efforts to obtain a census of the satellite populations around such hosts (Chiboucas et al. 2009, 2013; Karachentsev et al. 2014, 2015; Merritt et al. 2014; Crnojević et al. 2016, 2019; Javanmardi et al. 2016; Bennet et al. 2017; Müller et al. 2017; Smercina et al. 2018). One focus is to measure the satellite populations of Milky-Way-like hosts (Javanmardi et al. 2016; Geha et al. 2017) in order to constrain cosmic variance among halos of similar mass (Moster et al. 2011; Fielder et al. 2019). In this context, the Satellites Around Galactic Analogues survey (SAGA; Geha et al. 2017) has revealed a population of star-forming satellites which project within the virial radii of Milky-Way-like hosts. The ongoing star formation in the bulk of the SAGA detections stands in contrast to the largely quiescent, gas-poor satellite population of the Milky Way itself (e.g., McConnachie 2012; Spekkens et al. 2014). This raises the possibility that satellite gas stripping and star formation quenching proceed differently around halos of similar mass, providing an important new observational constraint on the underlying physics (e.g., Wetzel et al. 2015; Fillingham et al. 2018; Simpson et al. 2018; Garrison-Kimmel et al. 2019).

The nearby Milky-Way-like spiral M101 $\left(D_{\mathrm{M} 101}=7.0 \mathrm{Mpc}\right.$, $V_{\mathrm{M} 101}=241 \mathrm{~km} \mathrm{~s}^{-1}$ adopted in this work; Lee \& Jang 2012;
Mihos et al. 2013; Tikhonov et al. 2015) has been the target of several LSB searches aiming to characterize its satellite populations (Karachentsev et al. 2014, 2015; Merritt et al. 2014; Javanmardi et al. 2016; Bennet et al. 2017; Müller et al. 2017). Many of the detected LSB objects have morphologies consistent with satellites of that host, although their asymmetric sky distribution is challenging to explain if they are all bona fide companions (Bennet et al. 2017).

This tension is alleviated somewhat by the presence of a background group in the vicinity of M101 $\left(D_{\mathrm{BG}} \sim 27 \mathrm{Mpc}, V_{\mathrm{BG}} \sim 1961 \mathrm{~km} \mathrm{~s}^{-1}\right.$; Tully 2015; Merritt et al. 2016), which includes the massive ellipticals NGC 5485 and NGC 5473 among its 25 members (Saulder et al. 2016; Karachentsev \& Makarova 2019). Indeed, followup observations to characterize the properties of the LSB dwarf candidates around M101 suggest that many lie at the distance of the NGC 5485 group (Merritt et al. 2016; Bennet et al. 2019; Carlsten et al. 2019). The distances to many others remain unconstrained, and there is a need for additional follow-up observations to constrain both their locations and their physical properties.

Deep searches for the atomic hydrogen (H I) reservoirs of LSB detections are a powerful tool for constraining their physical properties (Spekkens \& Karunakaran 2018); detections provide estimates of distance and HI mass, while nondetections yield upper limits on the gas richness of objects along the line of sight (LOS). Since the H I content of field dwarfs (Huang et al. 2012; Bradford et al. 2015) as well as the environmental dependence of that content (Grcevich \& Putman 2009; Spekkens et al. 2014; Brown et al. 2017) are well characterized in the local universe, H I follow-up observations provide a mechanism for constraining the physical properties of the LSB dwarf candidates and the impact of their environments on those properties. 
We present $\mathrm{H}$ I follow-up observations along the LOS to 27 LSB dwarf candidates in the M101 region using the Robert C. Byrd Green Bank Telescope (GBT). We aim to characterize their gas properties and to use that information to place the M101 and NGC 5485 systems into context with other Milky Way-like hosts and nearby galaxy groups.

The structure of this paper is as follows. In Section 2, we describe our H I target selection. We outline our observations and data reduction procedure in Section 3. In Section 4, we present the properties of our detections as well as upper limits on the gas content of our nondetections. In Section 5, we discuss the implications of these findings for the membership of the M101 and NGC 5485 group systems, and for the influence of these hosts on the gas content of the satellites. We summarize in Section 6.

\section{Sample Selection}

The M101 satellite system consists of four previously known, more luminous satellites $\left(M_{V}<-14\right.$ : NGC 5474, NGC 5477, Holm IV, and DDO 194; Tikhonov et al. 2015). In their HI mapping study of the M101 region, Mihos et al. (2012) detected two low-mass H I features. We do not consider these in our sample selection; one does not have an optical counterpart, while the other has a recessional velocity that is not consistent with M101. Recent optical LSB searches, described in the previous section, have identified fainter satellite candidates (five of which have been recently confirmed with $M_{V}<-8.2$ : DF-1, DF-2, DF-3, DwA, Dw9; Danieli et al. 2017; Bennet et al. 2019, see below). As described below, we select our sample of $\mathrm{H}$ I targets from M101 satellite candidates identified in those LSB searches.

Merritt et al. (2014, hereafter M14) carried out one of the first modern LSB searches around M101 using the Dragonfly Telephoto Array, discovering seven dwarf candidates in a $\sim 9 \mathrm{deg}^{2}$ field. In their survey around nearby spirals, Karachentsev et al. (2015, hereafter K15) discovered an additional four dwarf candidates in the region around M101, one of which (DwA/DGSAT1) was separately discovered by Javanmardi et al. (2016). LSB searches were also conducted using extant data. Bennet et al. (2017, hereafter B17) confirmed the M14 and K15 detections and discovered 38 new LSB dwarf candidates in a $\sim 9 \mathrm{deg}^{2}$ field around M101 in the Canada France Hawaii (CFHT) Legacy Survey data using a semiautomated algorithm tuned to reveal LSB features. While the preceding searches focused in the immediate region around M101, Müller et al. (2017, hereafter M17) searched through SDSS data for new LSB dwarf candidates over $330 \mathrm{deg}^{2}$ in the broader M101 group complex to reveal six new candidates beyond the M101 virial radius. In combination, these four studies revealed a total of 55 unique LSB dwarf candidates in the region around M101.

We select all LSB dwarf candidates from the combined samples of M14, K15, B17, and M17 with apparent magnitudes of $m_{V}<19.5$ mag for H I follow-up with the GBT. The optical properties of the resulting 27 targets are given in Table 1. Since we determine observing times from a gas richness scaling relation for local dwarfs (Bradford et al. 2015; see Section 3), our adopted $m_{V}$ threshold implies a follow-up time for each target of a few hours at most (see column 10 of Table 1). Column 7 of Table 1 gives the references for the optical properties that we adopt in this study.
A variety of studies have constrained distances to the M101 LSB dwarf candidates since their discovery, and columns 8 and 9 of Table 1 list the value that we adopt for our follow-up targets. Hubble Space Telescope (HST) campaigns reported by Merritt et al. (2016, hereafter M16), Danieli et al. (2017, hereafter D17), and Bennet et al. (2019, hereafter B19) have either confirmed a dwarf candidate's association with M101 from tip of the Red Giant Branch (TRGB) distances from resolved star color-magnitude diagrams, or reported lower distance limits derived from the lack of resolved stars. Distance constraints are also reported by Carlsten et al. (2019, hereafter C19) via a surface brightness fluctuation (SBF) technique. We adopt distances or lower limits from M16, D17, or B19 when available, and otherwise we adopt the C19 estimates. Table 1 shows that of the 27 M101 dwarf candidates that we targeted four have distance estimates consistent with M101, 18 have distance lower limits that place them in the M101 background, and the remaining five have no prior distance information.

We note that although a combination of stellar mass and color is a more accurate predictor of gas content than stellar mass alone for the high surface brightness galaxy population (e.g., Catinella et al. 2012; Brown et al. 2015), we do not use $g-r$ as a selection criterion for our H I follow-up sample. Instead, we investigate the utility of the available colors for LSB dwarf candidates as a predictor of gas richness in Section 5 .

\section{Observations and Data Reduction}

We performed $63 \mathrm{hr}$ of observations between 2016 August and 2019 August using the GBT to determine the H I content along the LOS to the 27 LSB dwarf candidates in Table 1 (programs AGBT16B-046, AGBT17A-188, and AGBT17B235). We used the $L$-band receiver and the Versatile GBT Astronomical Spectrometer (VEGAS) with a bandpass of 100 $\mathrm{MHz}$ and spectral resolution of $3.1 \mathrm{kHz}$, dumping data every $5 \mathrm{~s}$ to build up the requisite integration time. This wide bandpass allows for the $\mathrm{HI}$ spectral line to be detected at heliocentric velocities up to $\sim 14,000 \mathrm{~km} \mathrm{~s}^{-1}$. This observational setup was used for all but one candidate, DF-3, which had a distance estimate that is consistent with that of M101 (D17) at the time it was observed. For this target, we used a narrower bandpass, $11.72 \mathrm{MHz}$, and a higher spectral resolution, $0.4 \mathrm{kHz}$, centered at the heliocentric velocity of $\mathrm{M} 101, V_{\mathrm{M} 101}=241 \mathrm{~km} \mathrm{~s}^{-1}$.

Our observations spent an equivalent amount of time on the target of interest (i.e., the "ON") and on a set of reference positions (that collectively constitute the "OFF"). We estimate the integration times for our targets using $m_{V}$ calculated from $m_{g}$ and $g-r$ following the relations in Jester et al. (2005) to reach a gas richness of $\frac{M_{\mathrm{HI}}}{L_{V}} \sim 1 \frac{M_{\odot}}{L_{\odot}}(\sim 0.5 \mathrm{dex}$ below the Bradford et al.( 2015 )scaling relations at $L_{V} \lesssim 10^{9} L_{\odot}$ ) with $\mathrm{S} / \mathrm{N}=5$ in a single $25 \mathrm{~km} \mathrm{~s}^{-1}$ channel. Gas richness is a distance-independent quantity since both $M_{\mathrm{H} \text { I }}$ and $L_{V}$ scale with distance squared. Therefore, a single spectrum allows us to meaningfully search for an $\mathrm{HI}$ reservoir in our targets anywhere within the wide bandpass.

The data were reduced using the standard GBTIDL $^{6}$ procedure getps. Before smoothing the raw spectra to our desired resolutions, we first removed narrowband and

\footnotetext{
http://gbtidl.nrao.edu/
} 
Table 1

Target LSB Dwarf Candidate Properties

\begin{tabular}{|c|c|c|c|c|c|c|c|c|c|c|c|}
\hline Name & $\begin{array}{c}\text { R.A. } \\
\text { H:M:S } \\
\text { (2) }\end{array}$ & $\begin{array}{l}\text { Decl. } \\
\text { D:M:S } \\
\text { (3) }\end{array}$ & $\begin{array}{c}m_{g} \\
(\mathrm{mag}) \\
(4)\end{array}$ & $\begin{array}{c}g-r \\
(\mathrm{mag}) \\
(5)\end{array}$ & $\begin{array}{c}r_{\text {eff }} \\
(\operatorname{arcsec}) \\
(6)\end{array}$ & $\begin{array}{c}\text { References } \\
\text { (7) }\end{array}$ & $\begin{array}{c}D_{\text {opt }} \\
(\mathrm{Mpc}) \\
(8)\end{array}$ & $\begin{array}{c}\text { References } \\
\text { (9) }\end{array}$ & $\begin{array}{c}\text { Int. Time } \\
\text { (hr) } \\
(10)\end{array}$ & $\begin{array}{c}\sigma_{25} \\
(\mathrm{mJy}) \\
(11)\end{array}$ & $\begin{array}{l}\text { H I } \\
\text { Det? } \\
(12)\end{array}$ \\
\hline$\overline{\mathrm{DF}-1}$ & $14: 03: 45.0$ & $53: 56: 40$ & $19.4 \pm 0.1$ & $0.60 \pm 0.14$ & $13.59 \pm 0.29$ & M16, B17 & $6.37_{-0.35}^{+0.35}$ & D17 & 1.4 & 0.49 & \\
\hline DF-2 & $14: 08: 37.5$ & $54: 19: 31$ & $19.8 \pm 0.1$ & $0.60 \pm 0.14$ & $10.35 \pm 0.68$ & M16, B17 & $6.87_{-0.30}^{+0.21}$ & D17 & 4.5 & 0.23 & \\
\hline DF-3 & $14: 03: 05.7$ & $53: 36: 56$ & $20.3 \pm 0.1$ & $-0.10 \pm 0.14$ & $20.0 \pm 2.6$ & M16, B17 & $6.52_{-0.27}^{+0.25}$ & D17 & 1.3 & 0.39 & \\
\hline DF-5 & $14: 04: 28.1$ & $55: 37: 00$ & $20.8 \pm 0.2$ & $0.20 \pm 0.28$ & $10.8 \pm 2.6$ & M16, B17 & $>17.5$ & M16 & 2.7 & 0.27 & $\mathrm{Y}$ \\
\hline DwA* & $14: 06: 49.8$ & $53: 44: 29$ & $19.5 \pm 0.1$ & $0.50 \pm 0.14$ & $10.92 \pm 0.23$ & K15, B17 & $6.83_{-0.26}^{+0.27}$ & B19 & 2.5 & 0.40 & \\
\hline DwB & $14: 08: 43.1$ & $55: 09: 57$ & $20.8 \pm 0.1$ & $0.80 \pm 0.22$ & $6.95 \pm 0.54$ & $\mathrm{~K} 15, \mathrm{~B} 17$ & $>15.1$ & B19 & 2.9 & 0.42 & Y \\
\hline DwC & $14: 05: 18.0$ & $54: 53: 56$ & $20.5 \pm 0.2$ & $0.70 \pm 0.28$ & $7.9 \pm 1.6$ & K15, B17 & $>15.1$ & B19 & 5.5 & 0.21 & \\
\hline DwD & $14: 04: 24.6$ & $53: 16: 19$ & $19.5 \pm 0.1$ & $0.30 \pm 0.14$ & $9.16 \pm 0.47$ & K15, B17 & $>15.1$ & B19 & 1.9 & 0.49 & \\
\hline Dw3 & $14: 08: 45.8$ & $55: 17: 14$ & $19.8 \pm 0.1$ & $0.50 \pm 0.22$ & $7.11 \pm 0.42$ & B17, B17 & $>15.1$ & B19 & 1.7 & 0.39 & \\
\hline Dw4 & $14: 13: 01.7$ & $55: 11: 16$ & $20.1 \pm 0.1$ & $0.20 \pm 0.14$ & $6.88 \pm 0.33$ & B17, B17 & $>15.1$ & B19 & 3.6 & 0.25 & \\
\hline Dw5 & $14: 04: 13.0$ & $55: 43: 34$ & $20.2 \pm 0.2$ & $0.10 \pm 0.22$ & $7.64 \pm 0.86$ & B17, B17 & $>15.1$ & B19 & 5.7 & 0.22 & \\
\hline Dw6 & $14: 02: 20.1$ & $55: 39: 17$ & $19.9 \pm 0.1$ & $0.50 \pm 0.14$ & $8.34 \pm 0.37$ & B17, B17 & $>15.1$ & B19 & 1.6 & 0.38 & \\
\hline Dw7 & $14: 07: 21.0$ & 55:03:51 & $21.1 \pm 0.1$ & $1.70 \pm 0.14$ & $4.7 \pm 0.20$ & B17, B17 & $>15.1$ & B19 & 4.4 & 0.29 & \\
\hline Dw8 & $14: 04: 24.9$ & $55: 06: 13$ & $19.8 \pm 0.1$ & $0.50 \pm 0.14$ & $5.70 \pm 0.20$ & B17, B17 & $>15.1$ & B19 & 1.6 & 0.34 & \\
\hline Dw13 & 14:08:01.2 & $54: 22: 30$ & $20.4 \pm 0.1$ & $0.60 \pm 0.14$ & $3.91 \pm 0.1$ & B17, B17 & $>15.1$ & B19 & 3.6 & 0.29 & \\
\hline Dw19 & 14:10:20.1 & $54: 45: 50$ & $20.4 \pm 0.1$ & $0.60 \pm 0.14$ & $4.56 \pm 0.23$ & $\mathrm{~B} 17, \mathrm{~B} 17$ & $>10.2$ & C19 & 5.4 & 0.35 & \\
\hline Dw26 & 14:08:50.4 & $53: 27: 24$ & $20.2 \pm 0.1$ & $0.40 \pm 0.14$ & $5.08 \pm 0.10$ & B17, B17 & $\cdots$ & $\cdots$ & 3.4 & 0.29 & $\mathrm{Y}$ \\
\hline Dw31 & $14: 07: 41.7$ & $54: 35: 18$ & $19.4 \pm 0.1$ & $0.60 \pm 0.14$ & $5.91 \pm 0.23$ & B17, B17 & $>10.8$ & $\mathrm{C} 19$ & 1.0 & 0.61 & \\
\hline Dw32 & $14: 07: 46.4$ & $54: 15: 26$ & $19.0 \pm 0.1$ & $0.20 \pm 0.14$ & $10.70 \pm 0.25$ & B17, B17 & $>12.6$ & C19 & 1.0 & 0.76 & \\
\hline Dw33 & $14: 08: 33.8$ & $55: 26: 49$ & $19.8 \pm 0.1$ & $0.60 \pm 0.22$ & $4.59 \pm 0.21$ & B17, B17 & $18.6_{-2.9}^{+3.4}$ & C19 & 2.0 & 0.47 & \\
\hline Dw38 & $14: 01: 17.6$ & $54: 21: 14$ & $20.1 \pm 0.1$ & $0.30 \pm 0.14$ & $4.33 \pm 0.16$ & B17, B17 & $>26.3$ & C19 & 3.3 & 0.62 & \\
\hline dw1343+58 & $13: 43: 07$ & $58: 13: 40$ & $15.54 \pm 0.30$ & $0.37 \pm 0.42$ & $28.6 \pm 1.3$ & M17, M17 & $\ldots$ & $\ldots$ & 0.2 & 2.35 & $\mathrm{Y}$ \\
\hline dw1355+51 & $13: 55: 11$ & $51: 54: 29$ & $18.76 \pm 0.30$ & $0.67 \pm 0.42$ & $7.44 \pm 1.3$ & M17, M17 & $\ldots$ & $\ldots$ & 0.5 & 0.79 & \\
\hline dw1408+56 & 14:08:41 & $56: 55: 38$ & $18.01 \pm 0.30$ & $0.51 \pm 0.42$ & $11.0 \pm 1.3$ & M17, M17 & $11.2_{-2.4}^{+3.4}$ & $\mathrm{C} 19$ & 0.2 & 0.52 & $\mathrm{Y}$ \\
\hline dw1412+56 & $14: 12: 11$ & $56: 08: 31$ & $19.46 \pm 0.30$ & $0.71 \pm 0.42$ & $8.08 \pm 1.3$ & M17, M17 & $>9.0$ & $\mathrm{C} 19$ & 0.7 & 0.52 & \\
\hline dw1416+57 & $14: 16: 59$ & $57: 54: 39$ & $19.06 \pm 0.30$ & $0.23 \pm 0.42$ & $7.35 \pm 1.3$ & M17, M17 & $\cdots$ & $\cdots$ & 0.8 & 0.66 & \\
\hline dw1446+58 & $14: 47: 00$ & $58: 34: 04$ & $18.46 \pm 0.30$ & $0.56 \pm 0.42$ & $8.97 \pm 1.3$ & M17, M17 & $\cdots$ & $\cdots$ & 0.3 & 0.85 & \\
\hline
\end{tabular}

Note. Column (1): adopted LSB dwarf candidate names. Columns (2) and (3): J2000 position of an optical centroid, which corresponds to our GBT LOS. Columns (4) and (5): $g$-band apparent magnitudes and $g-r$ colors. Column (6): effective radius of the LSB dwarf candidate. Column (7): first is the reference list of the origin of the candidate name and position in columns (1)-(3); the second lists the source of the photometric measurements in column. (4)-(6). Column (8): adopted distance constraints from the references in column (9). Column (10): total effective GBT integration time, including the ON+OFF positions and subtracting any time lost due to RFI flagging. Column (11): representative rms noise of the spectrum at a velocity resolution of $\Delta V=25 \mathrm{~km} \mathrm{~s}^{-1}$. Column (12): flag indicating whether or not we detect H I emission associated with a dwarf candidate. References: M14, Merritt et al. (2014); K15, Karachentsev et al. (2015); M16, Merritt et al. (2016); B17, Bennet et al. (2017); D17, Danieli et al. (2017); M17, Müller et al. (2017); B19, Bennet et al. (2019); and C19, Carlsten et al. (2019). *Target initially reported by K15 but the GBT LOS corresponds to a position from Javanmardi et al. (2016); given the 9!1 GBT beam, an offset of a few arcseconds is negligible.

broadband radio frequency interference (RFI). The latter is primarily present as a strong, intermittent GPS signal at $\nu \sim 1.38 \mathrm{GHz}$ that is best removed by flagging the entire $5 \mathrm{~s}$ data dumps in which the RFI is present. On average, we flagged $20 \%$ of the data to remove this feature. Narrowband RFI presents itself throughout the entire spectrum as spurious, strong signals that span a few raw spectral channels. As scans and their constituent integrations are co-added and eventually smoothed, these narrowband features may resemble the expected H I profile of a dwarf if not excised. To remove narrowband RFI, we searched through all channels of each integration for signals that exceed five times the median absolute deviation. This threshold value was found to be the most effective at identifying RFI spikes while avoiding noise fluctuations. The values in these channels were then replaced with the median of the 200 surrounding channels.

The calibrated, RFI-excised spectra were smoothed to multiple resolutions from 5 to $50 \mathrm{~km} \mathrm{~s}^{-1}$ and examined by eye to search for statistically significant emission. A representative rms noise for each spectrum at $\Delta V=25 \mathrm{~km} \mathrm{~s}^{-1}$ resolution is given in column 11 of Table 1 . We detect $\mathrm{HI}$ emission along the LOS to five targets (column 12 of Table 1).
Their spectra are shown in Figure 1 at $\Delta V$ given in Table 2, which lists all other properties derived from these $\mathrm{HI}$ detections. We find no emission associated with the 22 remaining targets. These spectra, with velocity resolution, $\Delta V=25 \mathrm{~km} \mathrm{~s}^{-1}$, are shown in Figure 2, and the corresponding upper limits on H I mass and gas richness are in Table 3.

\section{Results \\ 4.1. H I Detections}

We detect $\mathrm{HI}$ along the LOS to five of the LSB dwarf candidates in our sample, and their spectra are shown in Figure 1. At our observing frequency of $\sim 1.4 \mathrm{GHz}$, the FWHM of the GBT beam, 9!1, encompasses the entire stellar component of our dwarf targets. The GBT beam response is well understood down to $\approx-30 \mathrm{~dB}$ (e.g., Spekkens et al. 2013) and we can assess the extent to which gas-rich sources near the LOS contaminate our spectra. For all of our targets, we search through $\mathrm{NED}^{7}$ and SDSS imaging catalogs (Aguado et al.

\footnotetext{
The NASA/IPAC Extragalactic Database (NED) is operated by the Jet Propulsion Laboratory, California Institute of Technology, under contract with the National Aeronautics and Space Administration.
} 


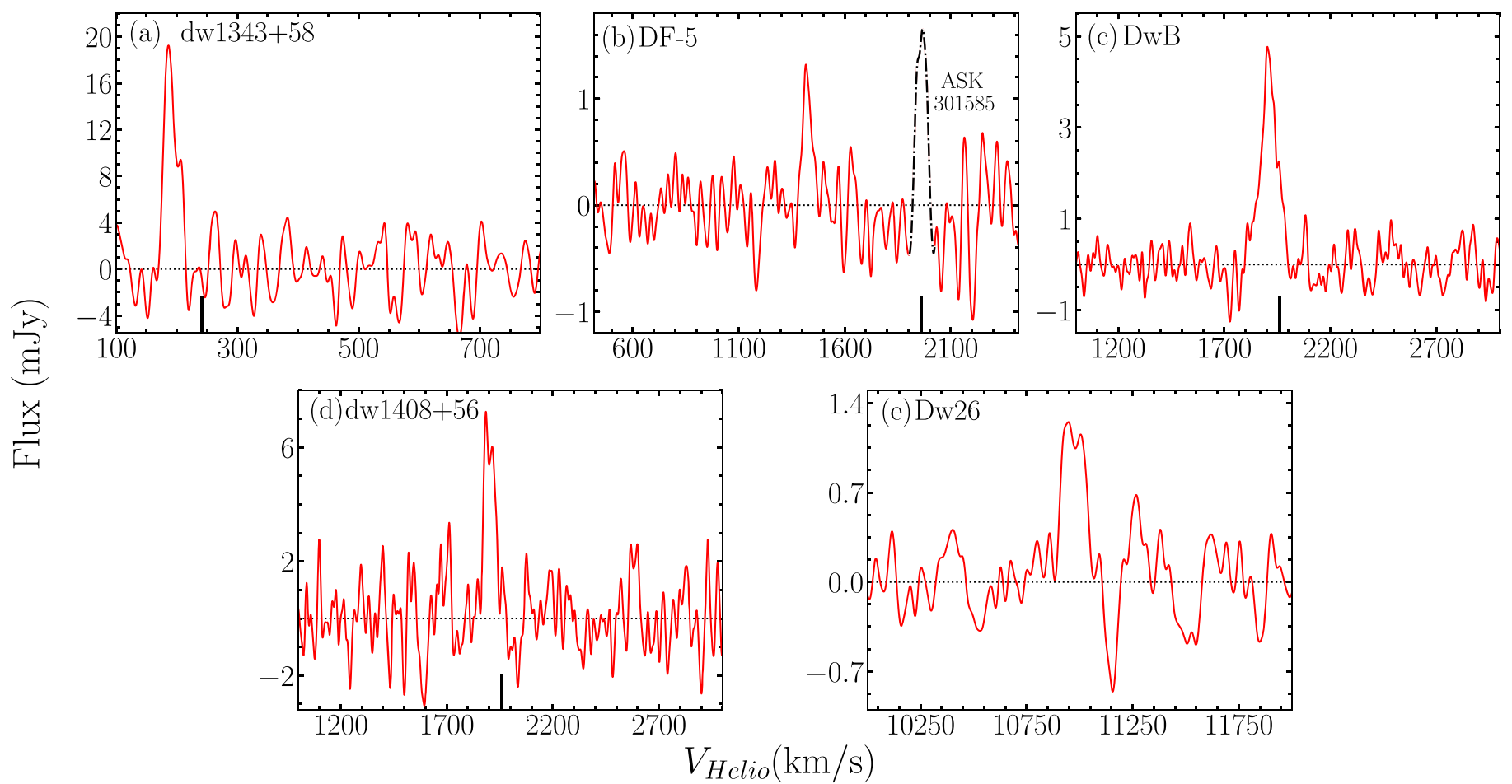

Figure 1. H I detections along the LOS to LSB dwarf candidates in the region around M101. Target names are in the top left corner of each panel and the spectral resolutions $\Delta V$ of the plotted spectra are in Table 2. The spectrum in (a) is off-center as Milky Way emission dominates at lower velocities. The dashed-dotted feature in (b) is the H I emission associated with ASK 301585 (see the text). The vertical black lines below the spectra indicate the $V_{\text {sys }}$ of M101 for (a) and the NGC 5485 group for (b)-(d). The derived properties of the H I detections are provided in Table 2.

2019) for objects within $30^{\prime}$ of the LOS that may present themselves as gas-rich interlopers in our spectra (the spectrum of one interloper, ASK 301585, is visible in panel (b) of Figure 1). We find no such interlopers for our H I detections and conclude that they are the H I counterparts to the LSB dwarf candidates along the LOS. The HI properties that we derive for the detections are given in Table 2.

We first derive distance-independent quantities, systemic velocities $\left(V_{\text {sys }}\right)$, and velocity widths $\left(W_{50}\right)$ following the methods of Springob et al. (2005). We fit first-order polynomials to both edges of each $\mathrm{HI}$ profile in Figure 1 between $15 \%$ and $85 \%$ of the peak flux value. To determine $V_{\text {sys }}$ and $W_{50}$, we first determine the velocities corresponding to the $50 \%$ flux level for each polynomial fit. The mean of these two values provides $V_{\text {sys }}$, while the difference provides $W_{50}$, which is also corrected for instrumental and cosmological redshift broadening to produce $W_{50, c}$. We do not correct for the effect of inclination on $W_{50}$. The uncertainties on the instrumental broadening correction, which we take to be $50 \%$, dominate those on $V_{\text {sys }}$ and $W_{50}$ (see Springob et al. 2005).

We estimate kinematic distances, $D_{\mathrm{H}}$, for DF-5, DwB, dw1408+56, and Dw26 using $V_{\text {sys }}$ of our detections and assuming $H_{0}=70 \mathrm{~km} \mathrm{~s}^{-1} \mathrm{Mpc}^{-1}$. The value of $D_{\mathrm{H} \text { I }}$ for DF-5 is consistent with the lower limits on $D_{\text {opt }}$ from M16 and C19. The values of $V_{\text {sys }}$ that we derive for DwB and dw1408+56 are similar to those of the background group containing NGC 5485, implying an association. The SBF lower limit on $D_{\text {opt }}$ from $\mathrm{C} 19$ for DwB is consistent with our distance estimate, while their $D_{\text {opt }}$ estimate for dw1408+56 is not. C19 note that their distance for this latter object may be unreliable due to its unusual morphology, and they conclude that it likely lies in the background of M101 as we find here. Our measurement of $D_{\mathrm{H} \text { I }}$ for Dw26 is the first distance estimate for this system (see also B17). For the purposes of this work, we assign a distance of $D_{\mathrm{H} \mathrm{I}}=D_{\mathrm{M} 101}=7.0 \mathrm{Mpc}$ to dw1343 +58 because of its similar recessional velocity to the broader M101 group complex.

We calculate the H I flux, $S_{\mathrm{H} \text { I }}=\int S \delta V$, by integrating over the line profile. H I masses, $M_{\mathrm{H} \text { I }}$, are determined using the standard equation for an optically thin gas (Haynes \& Giovanelli 1984),

$$
M_{\mathrm{H} \mathrm{I}}=2.356 \times 10^{5}\left(D_{\mathrm{H} \mathrm{I}}\right)^{2} S_{\mathrm{H}} M_{\odot},
$$

where $D_{\mathrm{H} \mathrm{I}}$ is in megaparsecs and $S_{\mathrm{H} \mathrm{I}}$ is in $\mathrm{Jy} \mathrm{km} \mathrm{s}^{-1}$. Uncertainties are determined following the methods of Springob et al. (2005). Using $m_{g}$ and $g-r$ from Table 1, the relations of Jester et al. (2005), and $D_{\mathrm{H} \text { I }}$ we estimate the $V$ band luminosities, $L_{V}$, and the gas richnesses, $M_{\mathrm{H} \mathrm{I}} / L_{V}$. These values are tabulated in Table 2 .

\subsection{H I Nondetections}

We find no H I signal along the LOS to the remaining 22 LSB dwarf candidates in our sample that we can attribute to these objects. We show their spectra in Figure 2, normalized by the rms values $\sigma_{25}$ listed in Table 1 . We search through NED and SDSS imaging catalogs for potential interlopers within $30^{\prime}$ of our targets. In Figure 2, H I emission from nearby objects in the spectra are shown by green dashed lines with the object names given above them. We note that the features are positive if the corresponding object is located in the $\mathrm{ON}$ and negative if they are located in an OFF.

We use $\sigma_{25}$ to place single, $25 \mathrm{~km} \mathrm{~s}^{-1}$-channel $5 \sigma$ upper limits on the HI mass of the LSB dwarf candidate 
Table 2

Properties of LSB Dwarf Candidates with H I Detections

\begin{tabular}{|c|c|c|c|c|c|c|c|c|c|}
\hline Name & $\begin{array}{c}\Delta V \\
\left(\mathrm{~km} \mathrm{~s}^{-1}\right) \\
(2)\end{array}$ & $\begin{array}{c}\sigma_{\Delta V} \\
(\mathrm{mJy}) \\
(3)\end{array}$ & $\begin{array}{c}V_{s y s} \\
\left(\mathrm{~km} \mathrm{~s}^{-1}\right) \\
(4)\end{array}$ & $\begin{array}{c}W_{50, c} \\
\left(\mathrm{~km} \mathrm{~s}^{-1}\right) \\
(5)\end{array}$ & $\begin{array}{c}S_{\mathrm{H} \mathrm{I}} \\
\left(\mathrm{Jy} \mathrm{km} \mathrm{s}^{-1}\right) \\
(6)\end{array}$ & $\begin{array}{c}D_{\mathrm{H} \mathrm{I}} \\
(\mathrm{Mpc}) \\
(7)\end{array}$ & $\begin{array}{c}\log \left(L_{V}\right) \\
\left(\log \left[L_{\odot}\right]\right) \\
(8)\end{array}$ & $\begin{array}{c}\log \left(M_{\mathrm{H} \mathrm{I}}\right) \\
\left(\log \left[M_{\odot}\right]\right) \\
(9)\end{array}$ & $\begin{array}{l}\left(\frac{M_{\mathrm{HI}}}{L_{V}}\right) \\
\left(\frac{M_{\odot}}{L_{\odot}}\right) \\
(10)\end{array}$ \\
\hline DF5 & 20 & 0.3 & $1424 \pm 4$ & $36 \pm 6$ & $0.06 \pm 0.02$ & 20.3 & $6.26 \pm 0.08$ & $6.79 \pm 0.18$ & $3.4 \pm 1.5$ \\
\hline DwB & 15 & 0.4 & $1913 \pm 4$ & $69 \pm 5$ & $0.37 \pm 0.04$ & 27.3 & $6.65 \pm 0.04$ & $7.81 \pm 0.05$ & $14 \pm 2$ \\
\hline $\mathrm{dw} 1408+56$ & 15 & 1.0 & $1904 \pm 2$ & $55 \pm 4$ & $0.38 \pm 0.08$ & 27.2 & $7.70 \pm 0.12$ & $7.82 \pm 0.10$ & $1.3 \pm 0.4$ \\
\hline Dw26 & 30 & 0.2 & $10972 \pm 12$ & $81 \pm 16$ & $0.15 \pm 0.04$ & 156 & $8.32 \pm 0.04$ & $8.94 \pm 0.12$ & $4.2 \pm 1.3$ \\
\hline
\end{tabular}

Note. Column (2): velocity resolution of the spectrum used to compute the H I properties (see Figure 1). Column (3): rms noise of the spectrum at $\Delta V$ in column (2). Column (4): heliocentric systemic velocity. Column (5): velocity width of the $\mathrm{H}$ I detection, corrected for cosmological redshift and instrumental broadening. Column (6): integrated $\mathrm{H}$ I flux. Column (7): kinematic distance estimated using the Hubble-Lemaitre Law, $V_{\text {sys }}$, and $\mathrm{H}_{0}=70 \mathrm{~km} \mathrm{~s}^{-1} \mathrm{Mpc}^{-1}$, except for dw1343+58( ${ }^{*}$ ) for which the M101 distance is adopted; see the text for an explanation. Column (8): logarithm of the $V$-band luminosity calculated using $m_{g}$ and $g-r$ in Table 1 and $D_{\mathrm{H} \mathrm{I}}$ from column (7). Column (9): logarithm of the H I mass calculated from Equation (1) using $S_{\mathrm{H} \mathrm{I}}$ in column (6) and $D_{\mathrm{H} \text { I }}$ in column (7). Column (10): H I mass to $V$-band luminosity ratio (gas richness).

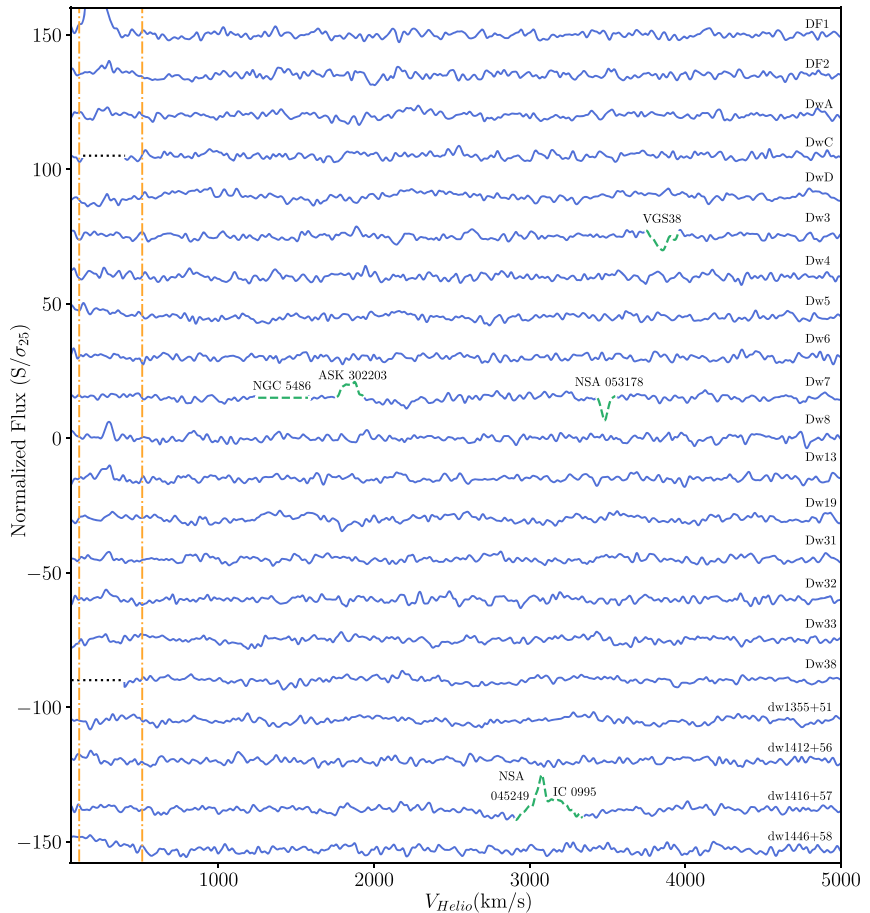

Figure 2. Spectra along the LOS to LSB dwarf candidates for which no associated $\mathrm{H} \mathrm{I}$ emission was found, at a resolution of $\Delta V=25 \mathrm{~km} \mathrm{~s}^{-1}$ and normalized by the rms values listed in Table 1 . The spectra are presented in the same order as in Table 3 but with DF-3 omitted because the spectral setup for that target was different; see the text for an explanation. Target names are shown above their respective spectrum at the right side of the figure. The vertical offset between the spectra is arbitrary to better display them. Parts of the spectra where an interloping $\mathrm{H}$ I signal of a nearby gas-rich galaxy is present in the ON or OFF scans are shown as green dashed lines. The vertical orange lines indicate the velocity region within which $\mathrm{H}$ I emission from M101 itself may be present. Two spectra (DwC and Dw38) have had M101 emission removed and replaced with black dotted lines for aesthetic purposes.

nondetections, $M_{\mathrm{H} \text { I }}^{\lim }$, using a modified version of Equation (1),

$$
M_{\mathrm{H} \text { I }}^{\lim }=2.945 \times 10^{7} D^{2}\left(\sigma_{25}\right) M_{\odot},
$$

where $\sigma_{25}$ is in $\mathrm{km} \mathrm{s}^{-1}$ and $D$ is in megaparsecs. For objects with $D_{\text {opt }}$ estimates or lower limits in Table 1, we use that value as $D$ in Equation (2). For objects lacking a $D_{\text {opt }}$ we compute $M_{\mathrm{H} \mathrm{I}}^{\lim }$ by setting $D$ to the M101 distance, $D_{\mathrm{M} 101}=7.0 \mathrm{Mpc}$. We also estimate the $\mathrm{H}$ I mass of these objects if they are located at
Table 3

H I Upper Limits for Nondetections

\begin{tabular}{lccc}
\hline \hline Name & $\begin{array}{c}\log \left(M_{\mathrm{HI}}^{\lim }\right) \\
\left(\log \left[M_{\odot}\right]\right)\end{array}$ & $\begin{array}{c}\log \left(M_{\mathrm{HI}}^{\lim }\right)_{\mathrm{BG}} \\
\left(\log \left[M_{\odot}\right]\right)\end{array}$ & $\begin{array}{c}\left(M_{\mathrm{HI}}^{\lim } / L_{V}\right) \\
\left(M_{\odot} / L_{\odot}\right)\end{array}$ \\
$(1)$ & $(3)$ & $\ldots$ & $(4)$ \\
\hline DF-1 & 5.77 & $\ldots$ & 0.70 \\
DF-2 & 5.51 & $\ldots$ & 0.49 \\
DF-3 & 5.69 & $\ldots$ & 1.86 \\
DwA & 5.75 & 6.67 & 0.68 \\
DwC & 6.16 & 7.02 & 0.81 \\
DwD & 6.52 & 6.93 & 0.91 \\
Dw3 & 6.43 & 6.73 & 0.87 \\
Dw4 & 6.22 & 6.68 & 0.84 \\
Dw5 & 6.18 & 6.92 & 0.88 \\
Dw6 & 6.41 & 6.80 & 0.92 \\
Dw7 & 6.29 & 6.87 & 1.12 \\
Dw8 & 6.36 & 6.81 & 0.75 \\
Dw13 & 6.30 & 6.88 & 1.08 \\
Dw19 & 6.04 & 7.12 & 1.27 \\
Dw31 & 6.33 & 7.22 & 0.89 \\
Dw32 & 6.55 & 7.00 & 0.94 \\
Dw33 & 6.69 & 7.13 & 0.99 \\
Dw38 & 7.11 & 7.23 & 2.01 \\
dw1355+51 & 6.06 & 7.05 & 0.61 \\
dw1412+56 & 6.58 & 7.15 & 0.74 \\
dw1416+57 & 5.98 & 7.26 & 0.85 \\
dw1446+58 & 6.09 & & 0.53 \\
\hline
\end{tabular}

Note. Column (2): $5 \sigma$ upper limit on $M_{\mathrm{H} \text { I }}$ calculated from Equation (2) using $D=D_{\text {opt }}$ and $\sigma_{25}$ from Table 1. Column (3): same as column (2) but instead $D=D_{\mathrm{BG}}=27 \mathrm{Mpc}$ of the NGC 5485 group for systems without $D_{\text {opt }}$ estimates or with lower limits. An exception is Dw33 which has a $D_{\text {opt }}$ estimate, however, we suggest it may reside in the background group (see Section 5.2). Column (4): upper limit on the gas richness (which is distanceindependent).

the distance of the NGC 5485 group, $\left(M_{\mathrm{H} \text { I }}^{\lim }\right)_{\mathrm{BG}}$, by setting $D=D_{\mathrm{BG}}=27 \mathrm{Mpc}$ in Equation (2). Both $M_{\mathrm{H} \mathrm{I}}^{\lim }$ and $\left(M_{\mathrm{H} \mathrm{I}}^{\lim }\right)_{\mathrm{BG}}$ are given in Table 3, along with the corresponding (distanceindependent) upper limit $M_{\mathrm{H} \text { I }}^{\lim } / L_{V}$ on gas richness.

\subsection{Optical and Gas Properties}

With the derived H I properties for our sample in hand we can make comparisons to their optical properties. Figure 3 plots $M_{\mathrm{H} \text { I }} / L_{V}$ for detections and $\left(M_{\mathrm{HI}}^{\lim }\right) / L_{V}$ for nondetections as a 


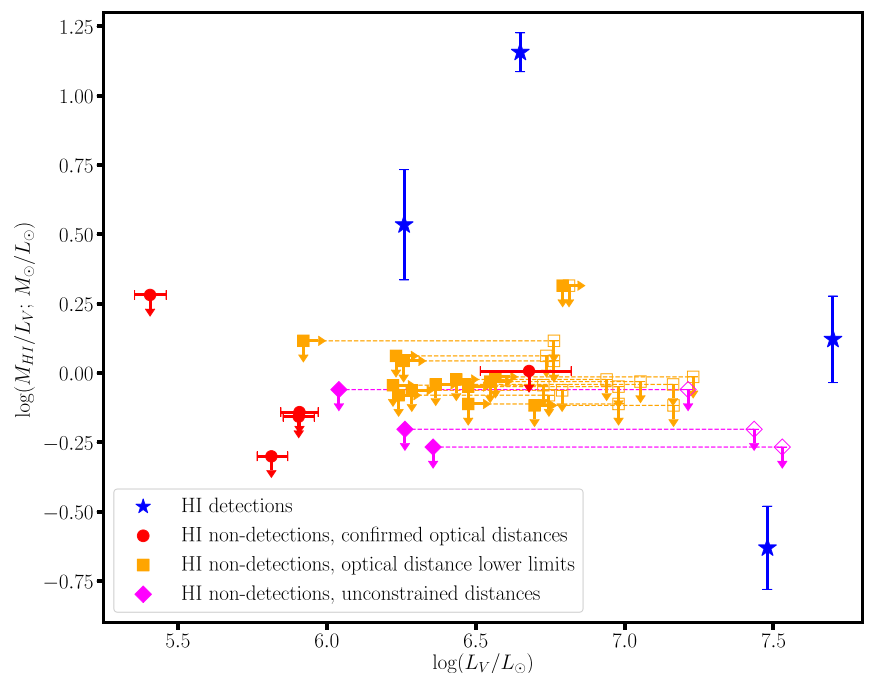

Figure 3. $M_{\mathrm{H} \mathrm{I}} / L_{V}$ (blue stars) and $M_{\mathrm{HI}}^{\lim } / L_{V}$ (red, orange, and magenta downward arrows) as a function of $L_{V}$ for LSB dwarf candidates in our sample. Among $\mathrm{H}$ I nondetections, red circles show objects with measured $D_{\text {opt }}$, orange squares show objects with lower limits on $D_{\text {opt }}$, and magenta diamonds show objects with no distance constraints. We place orange squares at $L_{V}$ corresponding to the $D_{\text {opt }}$ lower limits (filled symbol and rightward arrows) and $D_{\mathrm{BG}}$ (empty symbol) of the NGC 5485 group for the corresponding objects, and magenta diamonds at $L_{V}$ corresponding to $D_{\mathrm{M} 101}$ (filled) and $D_{\mathrm{BG}}$ (empty). Pairs of symbols for the same objects are connected by horizontal dashed lines. We omit one gas-rich target for aesthetic purposes, Dw26 (log $\left.\left(M_{\mathrm{H} \mathrm{I}} / L_{V}\right)=0.62, \log \left(L_{V}\right)=8.32\right)$.

function of $L_{V}$. We separate our sample into four groups: objects with $\mathrm{HI}$ detections (blue stars), nondetections with confirmed distances, $D_{\text {opt }}$ (red circles), nondetections with lower limits on $D_{\text {opt }}$ (orange squares), and nondetections with no distance measure (magenta diamonds). Since $M_{\mathrm{HI}} / L_{V}$ is distance-independent, the location of the points along the $y$-axis is known for all of the points. For nondetections with lower limits on $D_{\text {opt }}$ or no available estimates, we place symbols at both the $D_{\text {opt }}$ lower limit and $D_{\mathrm{BG}}$ (filled and empty orange squares) or $D_{\mathrm{M} 101}$ and $D_{\mathrm{BG}}$ (filled and empty magenta diamonds) along the $x$-axis and connect the two with a horizontal dashed line. For clarity, we do not include $\mathrm{HI}$ detection Dw26, with $\log \left(L_{V} ; L_{\odot}\right)=8.3$. Considering this object in addition to those plotted in Figure 3, over half of the H I detections correspond to objects with higher $L_{V}$ than the nondetections even if the latter are placed at the distance of the NGC 5485 group.

Figure 4 shows gas richness versus $g-r$ for the sample, separated into H I detections (blue stars) and H I nondetections (red circles with downward arrows). The horizontal dotted line indicates $M_{\mathrm{H} \mathrm{I}} / L_{V}=1 M_{\odot} / L_{\odot}$, which is typical for dwarf galaxies (e.g., Huang et al. 2012; Bradford et al. 2015). Both our detections and nondetections have a wide range of colors. We discuss this further in the following section.

\section{Discussion}

With the gas properties of our H I detections and nondetections in hand, we consider their most likely physical associations along with their implications for the gas richnesses of satellite systems on both the galaxy and group scales.

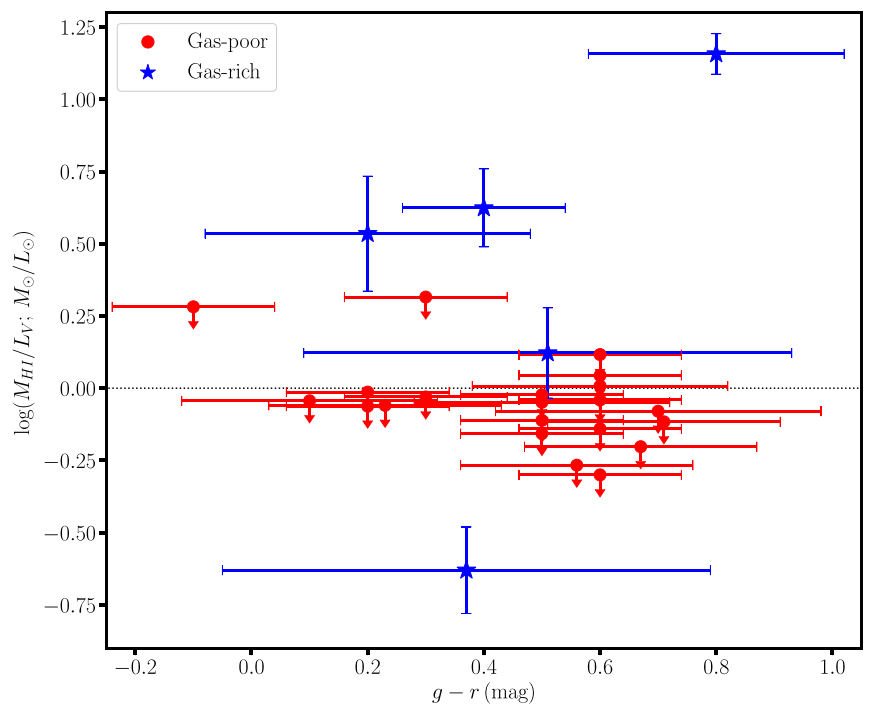

Figure 4. $M_{\mathrm{H} \mathrm{I}} / L_{V}$ (blue stars) and $M_{\mathrm{H} I}^{\lim } / L_{V}$ (red circles with downward arrows) vs. $g-r$ color for our sample. The horizontal dotted line shows $M_{\mathrm{HI}} / L_{V}=1 M_{\odot} / L_{\odot}$, typical for dwarf galaxies (e.g., Huang et al. 2012; Bradford et al. 2015). We omit one gas-poor outlier in color for aesthetic purposes, Dw7 $\left(\log \left(M_{\mathrm{HI}}^{\lim } / L_{V}\right)=0.05, g-r=1.7\right)$.

\subsection{Associations of H I Detections}

The systemic velocities of our five detections allow us to determine whether they are associated with M101 or the NGC 5485 group in the background (see Section 1), or whether they are at some other location along the LOS.

The systemic velocity of the H I detection of dw1343+58, $V_{\text {sys }}=195 \mathrm{~km} \mathrm{~s}^{-1}$ (Table 2), confirms that it is a nearby dwarf. Its projected separation from M101, $d_{\text {proj }} \sim 580 \mathrm{kpc}$, and similar $V_{\text {sys }}$ to $\mathrm{M} 101\left(V_{\mathrm{M} 101}=241 \mathrm{~km} \mathrm{~s}^{-1}\right)$ suggests that it is likely a member of the M101 group. The SDSS spectrum for $\mathrm{dw} 1343+58, V_{\mathrm{SDSS}}=165 \pm 33 \mathrm{~km} \mathrm{~s}^{-1}$, is consistent with the $V_{\text {sys }}$ that we derive, although the SDSS pipeline misclassified it as a star. Given its optical morphology, M17 suggest that $\mathrm{dw} 1343+58$ is a blue compact dwarf (BCD). The relatively low gas richness of dw1343+58 compared to typical starforming dwarfs (e.g., Huang et al. 2012; Bradford et al. 2015) and the UV counterpart that we identify in our search of archival Galaxy Evolution Explorer (GALEX) data provide further evidence in support of this classification (Thuan \& Martin 1981; Gil de Paz et al. 2003).

On the other hand, DwB and dw1408+56 have $V_{\text {sys }}$ consistent with that of the NGC 5485 group. Using their kinematic distances and projected separations, we estimate that the physical separations of DwB and dw1408+56 from NGC 5485 are $\sim 450 \mathrm{kpc}$ and $\sim 940 \mathrm{kpc}$, respectively. Given its kinematic distance, the properties of dw1408+56 resemble those of a gas-rich ultra-diffuse galaxy (UDG; $R_{\text {eff }}>1.5 \mathrm{kpc}, \mu_{0}>24 \mathrm{mag} \operatorname{arcsec}^{-2}$; van Dokkum et al. 2015) progenitor with $R_{\text {eff }}=1.45 \pm 0.17 \mathrm{kpc}$ and $\mu_{r, 0}=23.28 \pm 0.06 \mathrm{mag} \operatorname{arcsec}^{-2} \quad$ (Spekkens \& Karunakaran 2018). This finding is broadly consistent with UDG number-halo mass relation estimates (e.g., van der Burg et al. 2017), which imply that the number of UDGs in a group like that containing NGC 5485 should be of order 1 .

DF-5 was suggested to be a member of the NGC 5485 group since HST imaging does not resolve any stars (M16), and our $\mathrm{H}$ I detection confirms that it is in the background of M101 with $V_{\text {sys }}=1424 \mathrm{~km} \mathrm{~s}^{-1}$. While this $V_{\text {sys }}$ differs by $\sim 500 \mathrm{~km} \mathrm{~s}^{-1}$ 


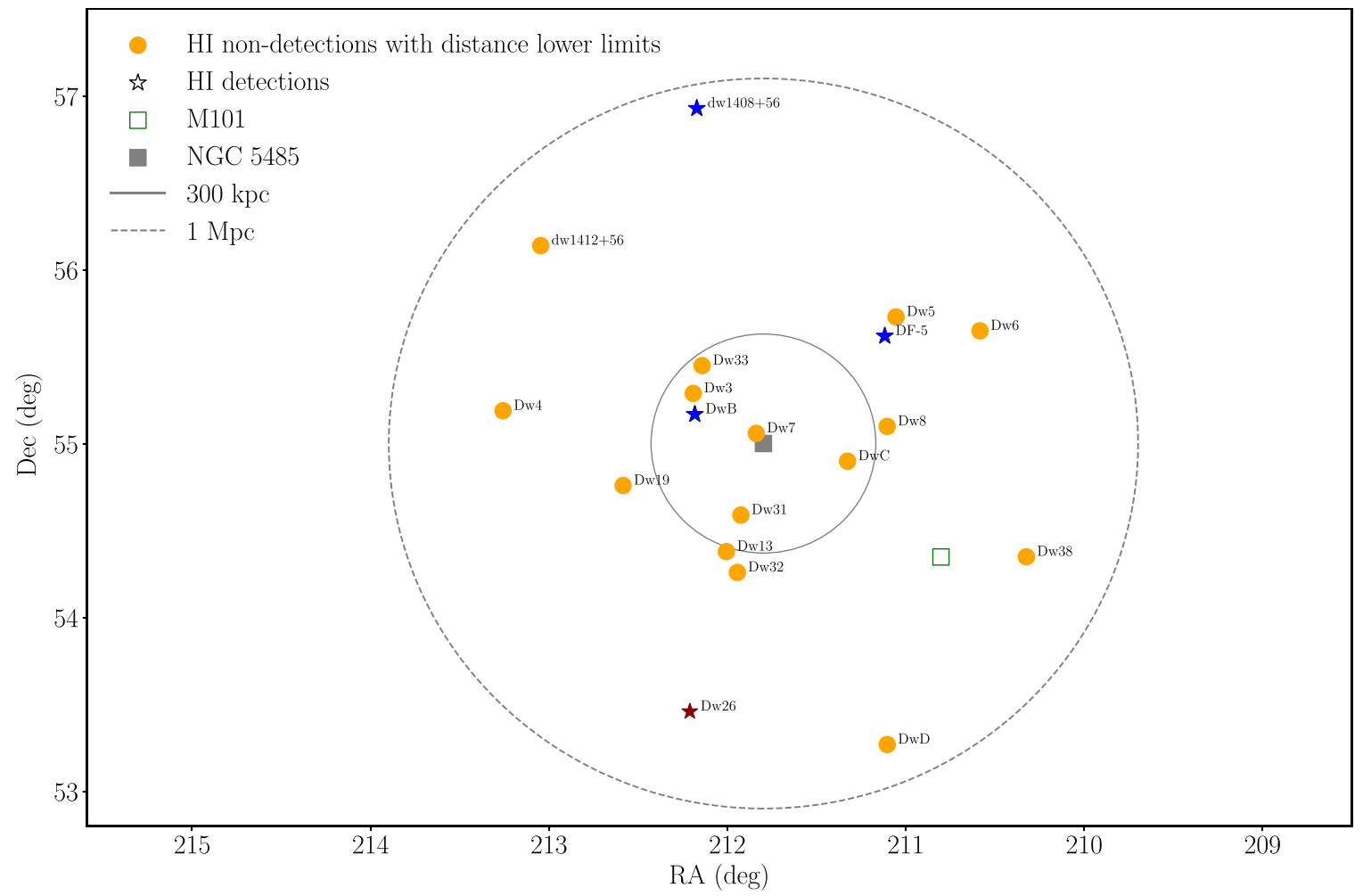

Figure 5. Targets in our sample that project within $\sim 2 \mathrm{deg}$ (=1 Mpc at the NGC 5485 distance; dashed circle) of NGC 5485 (solid gray square) and that have no known association with M101. Objects confirmed to be in the background of M101 via our H I detections are shown as stars: the filled in blue stars are those associated with the NGC 5485 group and the dark red star is Dw26 in the distant background. Objects that are undetected in H I but have optical distance measurements or lower limits that place them beyond M101 are shown as yellow circles. For reference, the open square shows the location of M101 in the foreground. The open gray circle centered on NGC 5485 has a radius of $300 \mathrm{kpc}$ at its distance, representative of literature estimates for the virial radius of the corresponding group (Tully 2015; Saulder et al. 2016; Karachentsev \& Makarova 2019).

from that of the group (Tully 2015), the small projected separation between DF-5 and NGC 5485 implies that a physical association is nonetheless feasible. We return to this issue in Section 5.2.

Dw26 is our most distant H I detection with $D_{\mathrm{H} \mathrm{I}}=156 \mathrm{Mpc}$. At such a distance, Dw26 is a star-forming galaxy well in the background of both M101 and the NGC 5485 group. The vast range of distances that we derive from our HI detections in the M101 region demonstrates the need for follow-up observations of optical LSB features to determine their physical properties and associations.

The relationship between stellar mass, color, and gas richness in the high surface brightness galaxy population is well studied (Catinella et al. 2012; Huang et al. 2012; Bradford et al. 2015; Brown et al. 2015): at a fixed stellar mass, bluer systems are more gas-rich. By contrast, Figure 4 illustrates that our $\mathrm{H}$ I detections exhibit the same broad range in $g-r$ as our nondetections, and therefore that the reported colors do not predict gas richness. While it is possible that star formation is regulated differently in these faint systems compared to the broader galaxy population (Wheeler et al. 2015; El-Badry et al. 2016; Di Cintio et al. 2019), we deem it more likely that the large photometric uncertainties at such low surface brightness (B17, M17), evidenced by the sizeable error bars in Figure 4, preclude the use of color as an indicator of the presence of gas. This further underscores the utility of follow-up H I observations to constrain the gas content and star formation activity in the LSB galaxy population.

\subsection{H I Nondetections and the NGC 5485 Group}

The majority of our follow-up H I observations resulted in nondetections, implying that the LSB dwarf candidates along those LOS are gas-poor. Among them, 15 out of 22 have distance constraints or lower limits from optical follow-up that place them in the background of M101 (see Table 1). Because field galaxies are almost universally star-forming and Hi-rich (Geha et al. 2012; Bradford et al. 2015) whereas our nondetections are clearly gas-poor, we consider the likelihood that all of our nondetections are associated with the NGC 5485 background group and have been stripped of their $\mathrm{H} \mathrm{I}$ reservoirs through environmental processes (e.g., Gatto et al. 2013; Wetzel et al. 2015; Simpson et al. 2018).

Figure 5 shows all of the targets in our sample that have no known association with M101 and project within $\sim 2$ degrees of NGC 5485 ( $=1 \mathrm{Mpc}$ at $D_{\mathrm{BG}}$; dashed circle). These targets are either confirmed to be in the background of M101 via our H I detections (stars) or are undetected in H I but have optical distance measurements or lower limits that place them beyond M101 (circles). Figure 5 suggests that, if our H I nondetections are at the NGC 5485 group distance and have been stripped of their gas due to environmental effects, then the sphere of influence of that group has a radius of at least $\sim 1 \mathrm{Mpc}$. Observations show that the fraction of gas-rich or star-forming satellites depends on the group mass (Brown et al. 2017; Schaefer et al. 2019), while simulations suggest that the environmental influence of a group extends beyond its virial radius, $R_{\mathrm{vir}}$ (Bahé et al. 2013; Cen et al. 2014). The latter suggest that the H I nondetections in Figure 5 could plausibly 
stem from gas stripping within $\sim 2-3 R_{\text {vir }}$ of the NGC 5485 group, which is broadly consistent with literature estimates of its $R_{\text {vir }}$ (solid circle in Figure 5; Tully 2015; Saulder et al. 2016; Karachentsev \& Makarova 2019).

Indeed, the properties of both our detections and nondetections are consistent with a scenario in which almost all are physically associated with the NGC 5485 group. In this picture, the nondetections correspond to galaxies that have had their gas stripped by the NGC 5485 intragroup medium or by tides, as the gas in satellite galaxies is not expected to survive after the first infall (Wetzel et al. 2015). The H I detections correspond to galaxies on the group outskirts that have not lost their gas or have yet to be processed by the group environment. NGC 5485 has stellar stream-like features which are likely due to past interactions (Karachentsev \& Makarova 2019), further supporting this scenario.

We consider each of the H I detections in turn to assess whether or not their properties are consistent with this scenario. The large projected separation between dw1408+56 and the group center as well as their correspondence in $V_{\text {sys }}$ suggest that this object is indeed on the group outskirts. Similarly, the large difference in $V_{\text {sys }}$ between DF-5 and the NGC 5485 group $\left(\sim 500 \mathrm{~km} \mathrm{~s}^{-1}\right)$ suggests that it is on its first infall with a large peculiar velocity. By contrast, DwB projects near the group center and has a similar $V_{\text {sys }}$; if it is indeed near the group center it should be gas-poor. This can be reconciled with our scenario if DwB is separated from the NGC 5485 group along the LOS. In this case, the correspondence between the NGC 5485 group $V_{\text {sys }}$ and that of DwB would stem from the peculiar velocity of the latter because it is on the first infall.

We note that the only $\mathrm{H}$ I follow-up target plotted in Figure 5 that is known not to be associated ${ }^{8}$ with the NGC 5485 group is Dw26, which our H I detection places far in the background $(D \sim 156 \mathrm{Mpc})$. However, it is unlikely that a significant number of our nondetections are at such large distances; a mechanism for stripping them in a lower density environment needs to be found. We therefore posit that the most plausible origin for the majority of our nondetections is that they belong to the NGC 5485 group.

\subsection{Satellite Gas Richness}

Our observations afford comparisons between the gas content of confirmed M101 satellites with that of the satellites of the Milky Way as well as with the populations around galaxies of similar mass. Figure 6 makes such a comparison: we plot $M_{V}$ as a function of projected separation, $D_{\text {proj }}$, at the host distance for all known M101 satellites (maroon symbols; Tikhonov et al. 2015; Danieli et al. 2017; Bennet et al. 2019) down to the B17 completeness limit of $M_{V}=-7.5$ (horizontal dotted line), all known Milky Way/Local Group satellites down to that same limit (yellow symbols; 2015 update of McConnachie 2012), and the 27 satellites detected within the projected virial radii of eight Milky Way mass hosts from the SAGA survey. The SAGA satellite populations are spectroscopically complete down to a limit of $M_{r}=-12.3$, or $M_{V}=-12.1$ for a median $g-r=0.4$ (horizontal dashed-dotted line; Geha et al. 2017). The stars denote

\footnotetext{
8 We note that the C19 SBF distance for Dw33 places it in the background of M101 but also in the foreground of NGC 5485. However, since C19 caution that the uncertainties on their Dw33 distance measurement may be underestimated, we deem it plausible that Dw33 is in fact associated with NGC 5485.
}

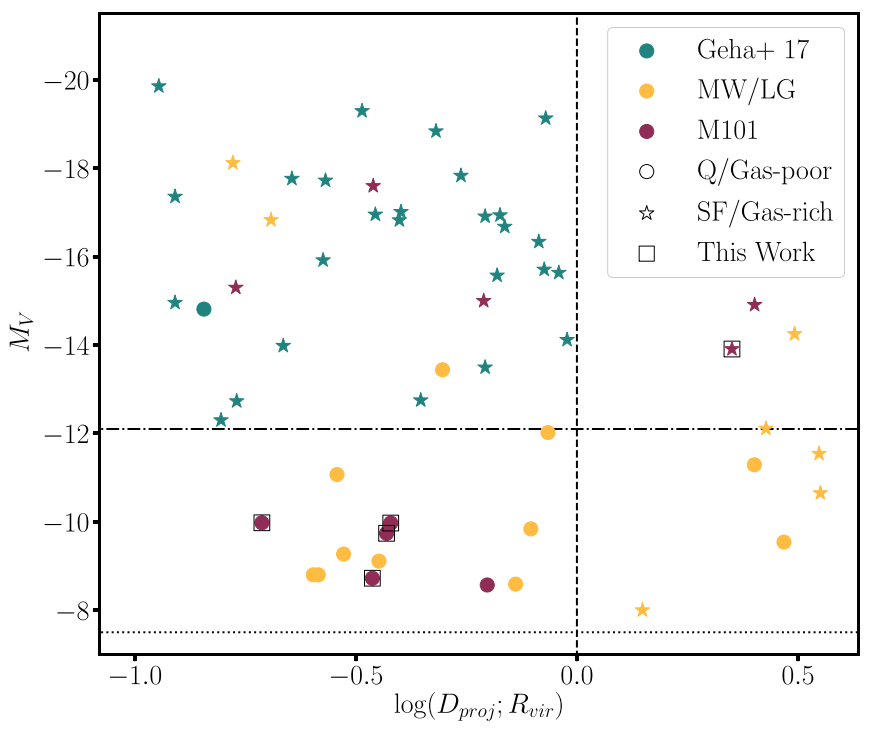

Figure 6. $M_{V}$ as a function of projected separation, $D_{\text {proj, }}$ at the host distance for all known M101 satellites (maroon symbols; Tikhonov et al. 2015; Danieli et al. 2017; Bennet et al. 2019) brighter than $M_{V}=-7.5$, all known Milky Way/Local Group satellites down to that same limit (yellow symbols; 2015 update of McConnachie 2012), and satellites brighter than $M_{V}=-12.1$ from the eight spectroscopically complete hosts from the SAGA survey (horizontal dashed-dotted line; Geha et al. 2017). Stars denote satellites that are starforming or gas-rich, and circles denote satellites that are quiescent or gas-poor. M101 satellites with H I observations from this work are enclosed by black boxes, and the vertical dashed line highlights $D_{\text {proj }}=R_{\text {vir }}$. We adopt $R_{\mathrm{vir}}=260 \mathrm{kpc}$ for M101 and $R_{\mathrm{vir}}=300 \mathrm{kpc}$ for the Milky Way and SAGA hosts.

satellites that are star-forming or gas-rich, and the circles denote satellites that are quiescent or gas-poor. M101 satellites with $\mathrm{HI}$ observations from this work are enclosed by black boxes, and the vertical dashed line indicates $D_{\text {proj }}=R_{\text {vir }}$. We adopt $R_{\mathrm{vir}}=260 \mathrm{kpc}$ for M101 (Merritt et al. 2014) and $R_{\mathrm{vir}}=300 \mathrm{kpc}$ for the Milky Way and the SAGA hosts (see Geha et al. 2017 for more detail).

Figure 6 illustrates that the satellites within $R_{\text {vir }}$ of M101, the Milky Way, or the SAGA hosts that are brighter than $M_{V} \simeq-12$ are typically star-forming or gas-rich, as are most companions beyond $R_{\mathrm{vir}}$. By contrast, the satellites within $R_{\mathrm{vir}}$ that are fainter than $M_{V} \simeq-12$ are typically quiescent or gaspoor. This difference in gas content and star formation activity between bright and faint satellites likely results from environmental effects: the gas reservoirs of low-mass subhalos are more easily stripped, and their star formation quenched, due to their shallower potential wells relative to high-mass subhalos (Wetzel et al. 2015; Emerick et al. 2016; Fillingham et al. 2016). Figure 6 therefore implies that the high fraction of starforming satellites detected within $R_{\text {vir }}$ by SAGA and the low fraction of H I-rich satellites within the $R_{\text {vir }}$ of M101 and the Milky Way can be explained by differences in survey sensitivity rather than inherent differences in the star formation activity among satellite populations. Instead we find that, when completeness is taken into account, the gas richnesses of the satellite populations of M101, the Milky Way, and the SAGA hosts within $R_{\text {vir }}$ are broadly consistent with one another. This suggests that the environment has a similar effect on the satellite populations of different Milky-Way-mass hosts, in line with expectations from simulations (e.g., Fillingham et al. 2018). 


\section{Conclusions}

We have presented $\mathrm{HI}$ follow-up observations using the GBT along the LOS to 27 optically detected LSB dwarf candidates in the M101 region (see Table 1). We find the H I counterparts of five targets (Figure 1) and derive their gas properties such as $\mathrm{HI}$ masses and velocity widths (Table 2). We find no $\mathrm{HI}$ emission along the LOS associated to the remaining 22 (Figure 2) and place stringent $5 \sigma$ single, $25 \mathrm{~km} \mathrm{~s}^{-1}$ channel upper limits on their H I masses and gas richnesses (Table 3).

Among the detections, we find that dw1343+58 is likely a member of the M101 group while Dw26 is far in the background with $D_{\mathrm{H}} \sim 150 \mathrm{Mpc}$. We find that the remaining three LSB dwarfs with HI detections (DF-5, DwB, and dw1408+56) are likely members of the background NGC 5485 group. We show that detections and nondetections span a similar range in measured color (Figure 4), implying that at these low surface brightnesses, measured colors are not a good indicator of gas content. These findings demonstrate the utility of H I follow-up of optically detected LSB features to constrain their physical properties.

The optical lower distance limits and projected separations of our H I nondetections from the elliptical galaxy NGC 5485 suggest that they all could be satellites of that group, where any gas they once had has been gas stripped by environmental effects (Figure 5). In this context, the sphere of influence of the NGC 5485 group has a radius of $R \sim 1 \mathrm{Mpc}$, in line with observational and theoretical constraints.

We compare the gas richnesses of confirmed M101 satellites to those of the Milky Way satellites and of similar mass hosts in the SAGA survey (Figure 6). Accounting for completeness, we find general agreement between these populations: satellites within the virial radius brighter than $M_{V} \simeq-12$ are broadly star-forming and gas-rich while those fainter than this threshold are broadly quiescent and gas poor. This suggests that the effect on satellite gas content is similar around hosts of similar stellar mass, in line with theoretical expectations.

We thank the anonymous referee for the useful comments that improved the clarity of this paper. K.S. acknowledges support from the Natural Sciences and Engineering Research Council of Canada (NSERC). Research by D.J.S. is supported by NSF grants AST-1821967, 1821987, 1813708, 1813466, and 1908972. Research by D.C. is supported by NSF grant AST-1814208, and by NASA through grants number HSTGO-15426.007-A and HST-GO-HST-GO-15332.004-A from the Space Telescope Science Institute, which is operated by AURA, Inc., under NASA contract NAS 5-26555.

The Green Bank Observatory is a facility of the National Science Foundation operated under cooperative agreement by Associated Universities, Inc.

Facility: GBT (VEGAS).

Software: astropy (Astropy Collaboration et al. 2013; PriceWhelan et al. 2018), GBTIDL.

\section{ORCID iDs}

A. Karunakaran (1) https://orcid.org/0000-0001-8855-3635

K. Spekkens (1) https://orcid.org/0000-0002-0956-7949

P. Bennet 난 https://orcid.org/0000-0001-8354-7279

D. J. Sand (1) https://orcid.org/0000-0003-4102-380X

D. Crnojević (ib) https://orcid.org/0000-0002-1763-4128
D. Zaritsky (1) https://orcid.org/0000-0002-5177-727X

\section{References}

Abraham, R. G., \& van Dokkum, P. G. 2014, PASP, 126, 55

Aguado, D. S., Ahumada, R., Almeida, A., et al. 2019, ApJS, 240, 23

Aihara, H., Arimoto, N., Armstrong, R., et al. 2018, PASJ, 70, S4

Astropy Collaboration, Robitaille, T. P., Tollerud, E. J., et al. 2013, A\&A, 558, A33

Bahé, Y. M., McCarthy, I. G., Balogh, M. L., \& Font, A. S. 2013, MNRAS, 430, 3017

Bennet, P., Sand, D. J., Crnojević, D., et al. 2017, ApJ, 850, 109

Bennet, P., Sand, D. J., Crnojević, D., et al. 2019, ApJ, 885, 153

Bradford, J. D., Geha, M. C., \& Blanton, M. R. 2015, ApJ, 809, 146

Brown, T., Catinella, B., Cortese, L., et al. 2015, MNRAS, 452, 2479

Brown, T., Catinella, B., Cortese, L., et al. 2017, MNRAS, 466, 1275

Carlsten, S. G., Beaton, R. L., Greco, J. P., \& Greene, J. E. 2019, ApJL, 878, L16

Catinella, B., Schiminovich, D., Kauffmann, G., et al. 2012, A\&A, 544, A65

Cen, R., Roxana Pop, A., \& Bahcall, N. A. 2014, PNAS, 111, 7914

Chiboucas, K., Jacobs, B. A., Tully, R. B., \& Karachentsev, I. D. 2013, AJ, 146,126

Chiboucas, K., Karachentsev, I. D., \& Tully, R. B. 2009, AJ, 137, 3009

Crnojević, D., Sand, D. J., Bennet, P., et al. 2019, ApJ, 872, 80

Crnojević, D., Sand, D. J., Spekkens, K., et al. 2016, ApJ, 823, 19

Danieli, S., van Dokkum, P., Merritt, A., et al. 2017, ApJ, 837, 136

Di Cintio, A., Brook, C. B., Macciò, A. V., Dutton, A. A., \& Cardona-Barrero, S. 2019, MNRAS, 486, 2535

El-Badry, K., Wetzel, A., Geha, M., et al. 2016, ApJ, 820, 131

Emerick, A., Mac Low, M.-M., Grcevich, J., \& Gatto, A. 2016, ApJ, 826, 148

Fielder, C. E., Mao, Y.-Y., Newman, J. A., Zentner, A. R., \& Licquia, T. C. 2019, MNRAS, 486, 4545

Fillingham, S. P., Cooper, M. C., Boylan-Kolchin, M., et al. 2018, MNRAS, 477, 4491

Fillingham, S. P., Cooper, M. C., Pace, A. B., et al. 2016, MNRAS, 463, 1916

Garrison-Kimmel, S., Wetzel, A., Hopkins, P. F., et al. 2019, MNRAS, 489,4574

Gatto, A., Fraternali, F., Read, J. I., et al. 2013, MNRAS, 433, 2749

Geha, M., Blanton, M. R., Yan, R., \& Tinker, J. L. 2012, ApJ, 757, 85

Geha, M., Wechsler, R. H., Mao, Y.-Y., et al. 2017, ApJ, 847, 4

Gil de Paz, A., Madore, B. F., \& Pevunova, O. 2003, ApJS, 147, 29

Grcevich, J., \& Putman, M. E. 2009, ApJ, 696, 385

Haynes, M. P., \& Giovanelli, R. 1984, AJ, 89, 758

Huang, S., Haynes, M. P., Giovanelli, R., \& Brinchmann, J. 2012, ApJ, 756,113

Javanmardi, B., Martinez-Delgado, D., Kroupa, P., et al. 2016, A\&A, 588, A89 Jester, S., Schneider, D. P., Richards, G. T., et al. 2005, AJ, 130, 873

Karachentsev, I. D., Bautzmann, D., Neyer, F., et al. 2014, arXiv:1401.2719

Karachentsev, I. D., \& Makarova, L. N. 2019, Ap, 62, 293

Karachentsev, I. D., Riepe, P., Zilch, T., et al. 2015, AstBu, 70, 379

Lee, M. G., \& Jang, I. S. 2012, ApJL, 760, L14

McConnachie, A. W. 2012, AJ, 144, 4

Merritt, A., van Dokkum, P., \& Abraham, R. 2014, ApJL, 787, L37

Merritt, A., van Dokkum, P., Danieli, S., et al. 2016, ApJ, 833, 168

Mihos, J. C., Harding, P., Spengler, C. E., Rudick, C. S., \& Feldmeier, J. J. 2013, ApJ, 762, 82

Mihos, J. C., Keating, K. M., Holley-Bockelmann, K., Pisano, D. J., \& Kassim, N. E. 2012, ApJ, 761, 186

Moster, B. P., Somerville, R. S., Newman, J. A., \& Rix, H.-W. 2011, ApJ, 731,113

Müller, O., Jerjen, H., \& Binggeli, B. 2018, A\&A, 615, A105

Müller, O., Scalera, R., Binggeli, B., \& Jerjen, H. 2017, A\&A, 602, A119

Price-Whelan, A. M., Sipőcz, B. M., Günther, H. M., et al. 2018, AJ, 156, 123

Saulder, C., van Kampen, E., Chilingarian, I. V., Mieske, S., \& Zeilinger, W. W. 2016, A\&A, 596, A14

Schaefer, A. L., Croom, S. M., Scott, N., et al. 2019, MNRAS, 483, 2851

Simpson, C. M., Grand, R. J. J., Gómez, F. A., et al. 2018, MNRAS, 478, 548

Smercina, A., Bell, E. F., Price, P. A., et al. 2018, ApJ, 863, 152

Spekkens, K., \& Karunakaran, A. 2018, ApJ, 855, 28

Spekkens, K., Mason, B. S., Aguirre, J. E., \& Nhan, B. 2013, ApJ, 773, 61

Spekkens, K., Urbancic, N., Mason, B. S., Willman, B., \& Aguirre, J. E. 2014, ApJL, 795, L5

Springob, C. M., Haynes, M. P., Giovanelli, R., \& Kent, B. R. 2005, ApJS, 160,149

Thuan, T. X., \& Martin, G. E. 1981, ApJ, 247, 823

Tikhonov, N. A., Lebedev, V. S., \& Galazutdinova, O. A. 2015, AstL, 41, 239 
Tully, R. B. 2015, AJ, 149, 171

van der Burg, R. F. J., Hoekstra, H., Muzzin, A., et al. 2017, A\&A, 607, A79 van Dokkum, P. G., Abraham, R., Merritt, A., et al. 2015, ApJL, 798, L45
Wetzel, A. R., Tollerud, E. J., \& Weisz, D. R. 2015, ApJL, 808, L27 Wheeler, C., Oñorbe, J., Bullock, J. S., et al. 2015, MNRAS, 453, 1305 Zaritsky, D., Donnerstein, R., Dey, A., et al. 2019, ApJS, 240, 1 